\title{
Gestión de los factores de riesgo vinculados al uso de Internet y las redes sociales en el estudiantado durante la etapa de la adolescencia en las organizaciones educativas
}

\author{
Management risk factors associated with the use of internet and social networks in the student during \\ adolescence in educational organizations
}

Recibido 03 enero 2013 • Aceptado 10 junio 2013 • Corregido 24 junio 2013

\author{
Wilbert Porras Quirós' \\ Universidad Nacional \\ Heredia, Costa Rica \\ willrony@yahoo.es
}

\begin{abstract}
Resumen. El uso la internet y las redes sociales es una temática que debe estar a la vanguardia del sistema educativo, por su auge en la actualidad y las consecuencias que tiene para el estudiantado en su proceso de formación. Por ello, se propone como objetivo para el presente escrito reflexionar acerca de las conductas de riesgo asociadas al uso la internet y las redes sociales en la población estudiantil, durante la etapa de adolescencia, llegando a la conclusión de la necesidad imperante, en el sistema educativo, de gestionar procesos de formación integral en donde se aborde esta temática como estrategia para fortalecer la calidad de vida del estudiantado.
\end{abstract}

Palabras claves. Educación, gestión, internet, redes sociales, identidad, adolescencia.

Abstract. The use of the internet and social networks is a topic that should be at the forefront of the educational system for its boom at present and the consequences for the students. Therefore, it is proposed as an objective for this paper to think about the risky behaviors associated with the use of the internet and social networks in students during the stage of adolescence, coming to the conclusion of the urgent need in the educational system to manage comprehensive training processes where this issue is addressed as a strategy to strengthen the quality of life of this population.

Keywords. Education, management, internet, social networks, identity, adolescence.

\footnotetext{
Bachiller en Orientación en la Universidad Nacional de Costa Rica. Experiencia en trabajo con adolescentes, madres, padres de familia y pescadores que viven en zonas rurales-costeras (Isla Venado, Isla Caballo y Cabuya) como asistente en el Área de Desarrollo Humano del Programa de Desarrollo Integral Comunitario Costero de la Universidad Nacional. Facilitador de cursos participativos en la Universidad Nacional.
} 


\section{Introducción}

Dentro de la gestión de la educación surge la necesidad de enfrentar adecuadamente, los retos producidos por los cambios tecnológicos de la actualidad. Si bien es cierto, grandes avances científicos como lo es la optimización de la Internet y su fácil acceso para casi toda la población estudiantil, es un gran apoyo para mejorar los procesos de enseñanza aprendizaje mediante la utilización de nuevas estrategias de mediación pedagógica, no se puede dejar de percibir los desafíos que conlleva su uso, pues existen una serie de riesgos vinculados a ello, los cuales son importantes de identificar y abordar dentro del sistema educativo.

La educación, en la actualidad, lleva la innegable misión de fortalecer en las personas las competencias que les permitan el análisis de sus contextos, con el fin de evaluar de él, aspectos positivos y negativos vinculados a las actividades realizadas en cada uno de los espacios con los que se cuenta, buscando "desarrollar conocimientos, valores, habilidades sociales y desarrollo de la inteligencia emocional" (Marambio, s.f., p. 1). Ante ello, si bien es cierto, la Internet viene a propiciar un gran aporte dentro de los nuevos paradigmas de la educación, se hace necesario conocer muchos de los peligros a los que se puede enfrentar el estudiantado al usarlo, sin ser conscientes de los riesgos a los cuales se ven expuestos si no se implementan las medidas preventivas acordes al caso.

En el presente escrito, se plantea la necesidad de reflexionar acerca de las conductas de riesgo asociadas con el uso de la Internet y las redes sociales en la población estudiantil, durante la etapa de adolescencia, como aspecto clave para gestionar, desde la educación, las herramientas fundamentales para que esta población se concientice de tomar medidas de precaución para el ingreso a este tipo de sitios dentro y fuera de las organizaciones educativas.

\section{Historia de la Internet}

La facilidad para el acceso a las nuevas tecnologías de la comunicación, posibilitan tener al mundo conectado entre sí. Esto es algo relativamente reciente que ha impactado de múltiples formas al sistema educativo costarricense (García, 2010a). La Internet tiene sus orígenes durante la segunda guerra mundial -aproximadamente en 1983- extendiendo su uso a los centros de estudio universitarios y posteriormente, a la población civil en general; ya para 1989, se fortalece con la invención del World Wide Web (www), haciendo posible -en 1991- el inicio del desarrollo de una comunicación mucho más interactiva.

En el año 1995, empieza el máximo apogeo, en donde casi cualquier persona que tenga un dispositivo para conectarse a este tipo de tecnología, lo puede hacer cada vez con mayor sencillez (Juncar, 2001), resaltándose esta facilidad, especialmente en las generaciones más 
jóvenes, quienes se han visto permeadas por los múltiples beneficios y amenazas que brindan las redes sociales, las cuales han sido todo un "boom" en la actualidad (Fernández, 2010).

Por su parte, aspectos tan tradicionales e importantes como son las relaciones humanas, han visto modificada su dinámica en relativamente pocos años, producto de la reestructuración acontecida por el uso de las tecnologías, propiamente en nuevos sistemas de comunicación. Hoy día, las nuevas generaciones de jóvenes llamadas por García (2010a) nativos digitales, son quizás, quienes se han visto mayormente afectadas con todos estos cambios, siendo partícipes de una fase de transición, en donde suelen carecer de guías adecuadas para formar las herramientas que les permita salir airosas de estas permutas, pues padres, madres y personal docente y administrativo en los centros educativos, no tienen la preparación para enfrentar los retos en el uso de las nuevas tecnologías de la comunicación, en las cuales, ya la formación de círculos de "amistades" trascienden fronteras, sin la necesidad de conocer de forma inmediata, quién es la otra persona con la cual se comparten situaciones e información personal (Álvarez, 2009).

Lo anterior, se refuerza con las estimaciones del Instituto Nacional de Estadísticas y Censos (INEC, 2010), en donde para ese año, hubo un total de 2,6 millones de personas entre 18 y 69 años, residentes en Costa Rica, de las cuales un 18,9\% pertenecía a una red social; es decir, aproximadamente 500,2 mil personas. Además, se resalta que el porcentaje de usuarios más alto, correspondía a hombres, personas con edad entre 18 y 29 años, con educación secundaria y universitaria, residentes en hogares de nivel medio y alto, de zonas urbanas. Contar con estos datos genera la necesidad de que en educación se tomen las medidas pertinentes al caso, generando la necesidad de mantenerse a la vanguardia del tema como parte de la realidad inmediata vivida por el estudiantado, lo que conlleva la creación de estrategias de gestión que contribuyan al uso adecuado y supervisado de la Internet y las redes sociales para el aprendizaje.

Ante este hecho, el sistema educativo costarricense tiene como reto actualizarse ante las nuevas corrientes propiciadas por un manejo de la comunicación más inmediato que en otros tiempos, dada en las redes sociales, en donde las personas educadoras podrían enfrentarse a la incertidumbre de no reconocer las ventajas y amenazas producidas en la población estudiantil, tras el uso de estos espacios, pues con ellos, se tiene acceso a múltiple información, así como a la conformación de grupos sociales que pueden tener consecuencias, positivas o negativas, según el conocimiento de alternativas para proteger la integridad de cada ser humano asumida tras su utilización. Aquí, es donde se hace necesario gestionar desde las diferentes casas de enseñanza, el fortalecimiento del pensamiento crítico para seleccionar, adecuadamente, con quién y qué se va a compartir en este tipo de sitios de la red.

Hoy día, desde el modelo educativo predominante, es necesario desarrollar las competencias necesarias en las personas profesionales encargadas de gestionar los procesos 
de formación del estudiantado para que estos últimos, logren desarrollar las capacidades de interrelacionar saberes y desarrollar relaciones humanas dignas, en las cuales se puedan aprender de ellas, los principios básicos (Marambio, s.f.) para lograr la autorrealización personal y grupal.

El fenómeno de las redes sociales, también se vivencia en el nivel internacional, donde las personas usuarias de la Internet, para el año 2000, era de trescientos sesenta millones, mientras que para el 2010, ya sobrepasaba los mil cuatrocientos millones (García, 2010a). Este mismo autor, menciona que el número de sitios de Internet, para 1993, eran ciento treinta; para el 2001, la cifra se había disparado hasta superar los treinta y un millones. En la actualidad, se calcula que hay más de cien millones, aumentando más las probabilidades de acceder a sitios desconocidos y altamente riesgosos para la población estudiantil, en general.

Al ser las redes sociales todo un fenómeno que ha emergido rápidamente, muchas veces se carece de las herramientas necesarias para utilizarlas de manera oportuna; es por ello, que se hace necesario gestionar esfuerzos en las organizaciones educativas para potenciar en el estudiantado las habilidades, conocimientos y competencias que les permita hacer un uso adecuado como una herramienta más para optimizar los procesos de enseñanza aprendizaje y no como agente potenciador de muchas amenazas, producto de una inadecuada utilización por la poca información que se posee en torno a los peligros a que se exponen en este tipo de sitios.

De ahí, que se requiera un proceso de inducción que se geste desde las políticas educativas, con el fin de prevenir situaciones de riesgo en el estudiantado con solo facilitarles información pertinente acerca de diferentes situaciones que les afectan. Por ello, se hace necesario abordar la gestión de los factores de riesgos relacionados con la utilización de la Internet como parte de un sistema de educación integral que debe prevalecer en la actualidad.

\section{Factores de riesgo vinculados con el uso de la Internet y las redes sociales}

Dentro de los factores de riesgo que se le presentan al estudiantado cuando ingresan al uso de la Internet y las redes sociales, especialmente durante la etapa de la niñez y la adolescencia, se encuentran: contar con pocas herramientas para seleccionar lo más apto para sí mismos y mismas, por aspectos propios de la etapa de desarrollo en la cual se encuentran, aunado a un auge social hacia la expansión económica, bienestar material y alto consumismo, en donde se ha relegado por los cambios socioculturales, aspectos tan fundamentales para el desarrollo humano, como son los valores y principios fundamentales que deben regir la convivencia consigo mismos y las demás personas. También se da, según García (2010b):

... el deterioro de ciertos valores humanos universalmente reconocidos como la intimidad, 
la empatía o la veracidad. Algunos de los comportamientos observados están fuera de la ley. Otros están afectando a cuestiones tan importantes como el rendimiento escolar o, incluso, el futuro laboral. (p. 41)

Además, se vincula a esta problemática: un aumento en hogares disfuncionales o en los cuales, se carece de la posibilidad de supervisar las actividades o contactos de los hijos e hijas, en redes sociales; la escasez de espacios libres y seguros, en donde los menores puedan ocupar su tiempo realizando actividades al aire libre (García, 2010a); profesionales de la educación con carencia parcial o absoluta para guiar a sus estudiantes en la uso de las tecnologías y las redes sociales, entre otros aspectos, los cuales generan la necesidad de desarrollar planes instituciones de educación fundamentados en la prevención, en donde se incite la reflexión crítica de la educación y la sociedad en general con el fin de incentivar una adecuada toma de decisiones con respecto a las actividades realizadas dentro de los diferentes espacios virtuales de Internet.

Para prevenir estos factores de riesgo, desde el sistema educativo se debe optar por una formación, en conjunto con el hogar, de valores y normas de convivencia humana en donde se sobreponga el bienestar personal y colectivo, haciendo consciente a los seres humanos de la riqueza, en cuestión de compartir criterios diversos, a los que se tiene alcance con el uso de las redes sociales pero aunado a ello, de los factores de riesgo a los cuales se exponen las personas si carece de las medidas preventivas para su utilización eficaz. Es aquí en donde se deben gestionar políticas educativas, en las cuales se capaciten constantemente a los y las profesionales vinculados a las Instituciones Educativas para que puedan compartir espacios de reflexión con sus estudiantes en los cuales incentiven en esta población, la reflexión acerca de su uso adecuado.

Cabe resaltar que existen múltiples factores de riesgo vinculados con el uso de las redes sociales e Internet, por ello, desde la administración de los sistemas educativos, se debe gestionar todo un plan preventivo para contrarrestar los riesgos que en estos espacios se puedan enfrentar, recordando que la educación es parte de una historia inclusa que se continúa escribiendo a lo largo de los años (Sander, s.f.), por ello su constante transformación y descubrimiento de nuevas necesidades sociales, las cuales son abordadas constantemente dentro de los contenidos curriculares con la finalidad de fortalecer en la población meta las competencias que les posibiliten una mejor calidad de vida.

\section{Redes sociales}

Hoy día, es casi una obligación ser partícipe de mínimo una red social, convirtiendo el acceso a este tipo lugares parte fundamental de la cotidianidad de muchas personas estudiantes, repercutiendo en ellas en dificultades para mantener la concentración durante tiempo lectivo, 
por la necesidad de conocer las nuevas actualizaciones de su grupo de amistades, quienes en muchos casos son sus propios compañeros y compañeras de salón. Luque (2008) define red social como:

(...) aquella que entra en la clasificación de "para establecer relaciones", su función principal es justamente eso relacionar las personas, y en principio cuantas más mejor. Esta filosofía, siendo aún la principal, no es la única ya que el software para una red social ha ido asumiendo todas las demás funciones como conversar, valorar, organizar y compartir. (p. 2)

Las exigencias construidas socialmente para ser parte de las redes sociales es bastante fuerte, aunado a la facilidad para lograrlo. Hoy día, existen múltiples promociones de dispositivos con acceso inmediato a Internet, tales como teléfonos, computadoras, tabletas, entre otros, con los cuales se debe convivir a diario en los espacios estudiantiles, en donde el profesional en educación debe enfrentarse a ellos, ya sea como un interruptor de los procesos educativos o como una herramienta más para el fortalecimiento de su quehacer. Generalmente, los espacios sociales virtuales si bien representan una amenaza para quienes los frecuentan, esto si no son utilizados con las medidas de precaución requeridas para tal caso, pueden a su vez ser, un incentivo para mediar procesos de formación integral en donde los jóvenes fomenten procesos de realimentación de la materia vista en clase, por ello, se requiere la actualización constante en su uso adecuado y constructivo como parte de la gestión educadora.

Las redes sociales son consideradas uno de los grandes avances de la actualidad, para Fernández (2010) "casi todos los jóvenes pertenecen al menos a una de ellas" Fernández (p. 9); son una gran fuente de misterio y aventuras, lugar en donde se suele producir -en quienes la frecuentan- fascinación y misticismo, casi similar a los vivenciados en los mismos cuentos de hadas. Esto se acompaña por el deseo de hallar el amor de la vida (o una ardiente pareja sexual) con quien compartir espacios de mutua interacción (Levis, 2001), aspectos que se deben discutir en el salón de clase, como parte de una tarea preventiva y fortalecedora de habilidades y competencias en el estudiantado, las cuales prevengan, mitiguen o eviten el desarrollo de valores negativos con el uso de dichas tecnologías en esta población.

Las redes sociales como espacios de interacción, suelen generar en el estudiantado, la flexibilidad suficiente, como para expresar los sentimientos y emociones más íntimas, de una forma anónima, sin temor de reproches o de posibles represarías por parte de personas conocidas. Además, permite interactuar, casi que en igualdad de condiciones, a la gran diversidad de población, sin importar rasgos físicos, creencias religiosas, edades y sin la necesidad de establecer vínculos afectivos, en fin, da la libertad para crear un perfil del prototipo de quien se es o el ideal imaginario de quien se quisiera ser; además, permite crear una identidad atractiva en la red (Bernárdez, 2006), aspecto que suelen Ilamar la atención para algunos adolescentes, los 
cuales, no tienen una autoestima adecuada o competencias sociales desarrolladas para hacerlo de manera inmediata, o porque simplemente les es más cómodo realizarlo de esta manera.

Aunado en lo anterior, la persona educadora tiene como reto, crear un ambiente de confianza, en donde el alumno o alumna vea al profesional en educación como a alguien a quien acudir en caso de necesitarlo, viendo en las redes un complemento y no un sustito para hacer catarsis o nuevos círculos de relaciones interpersonales. La educación es necesario que sea visionaria, que sea participante activa de un proceso de transformación de los métodos tradicionales de formación pero que conserve los rasgos más esenciales del humanismo, no se trata de ver a las redes sociales como un agente dañino, sino como un aliado más en la tarea de formación integral del estudiantado siempre y cuando se utilice adecuadamente, conociendo los riesgo que su uso puede generar.

Cabe destacar que en la actualidad, las redes sociales pueden ser vistas como un multifacético y amplio lugar de encuentro. También, un espacio para fortalecer las relaciones interpersonales de forma virtual, permitiendo con facilidad, conocer nuevas personas, charlar con "desconocidos", jugar, escapar de la rutina del trabajo y del estudio, así como en muchos casos, seducir y enamorarse (Levis, 2001; INEC, 2010). Las personas adolescentes, jóvenes y adultas, encuentran en las redes sociales, un lugar nutrido de actividades para realizar, en donde es indudable la participación del personal docente en conjunto con la familia, en la construcción de criterios aptos para disfrutarlos sanamente. Se hace importante, que desde los centros educativos se tome la batuta para fortalecer en las personas, habilidades y competencias enfocadas en la prevención y el cuidado de la integralidad.

Tanta facilidad ofrecida para ingresar a las redes sociales y conformar grupos de interacción, suele ser beneficioso para quienes tienen dificultad para socializar por timidez o por aspectos personales; sin embargo, a la vez, es una fuente de inminente peligro, especialmente cuanto se está la etapa de la adolescencia y no se cuenta con criterios de selección adecuados para discernir de los fines tenidos por aquellos sujetos con quienes se interactúa, peor aún, si se carece de figuras de apoyo, las cuales supervisen y conciencien acerca de los peligros de este tipo de sitios, en donde con frecuencia, se da información personal mediante texto, fotos, videos que les puede poner en inminente riesgo.

Cada día son más las personas pertenecientes a las redes sociales y consecuentemente, son mayores los peligros que emergen por dicha actividad, propiciando la generación de conductas de riegos en la población estudiantil, quienes por el momento de evolución tecnológica, son quienes suelen acceder con mayor facilidad (INEC, 2010), pero quienes a su vez, se exponen a más factores amenazantes, como por ejemplo, confiar excesivamente en lo que se les diga o se muestre en perfiles de identidad, fotos, videos, entre otros, poniendo en riesgo su propio ser e incluso a sus seres cercanos. 
Basándose en lo anterior, desde las Instituciones Educativas se deben reconocer lo suscitado anteriormente, promoviendo la concienciación en la población estudiantil el pensamiento crítico y los criterios de selección adecuados para elegir pertinentemente el tipo de actividades que se van a realizar dentro de las redes sociales, poniendo en evidencia que este tipo de lugares puede ser frecuentado por muchas personas con desconocidas intenciones las cuales les pueden perjudicar o beneficiar dependiendo de las medidas preventivas utilizadas por cada persona usuaria. La gestión de la educación en este sentido es fundamental que vaya en miras de crear la autonomía suficiente en el estudiantado para cuidar su propio bienestar integral.

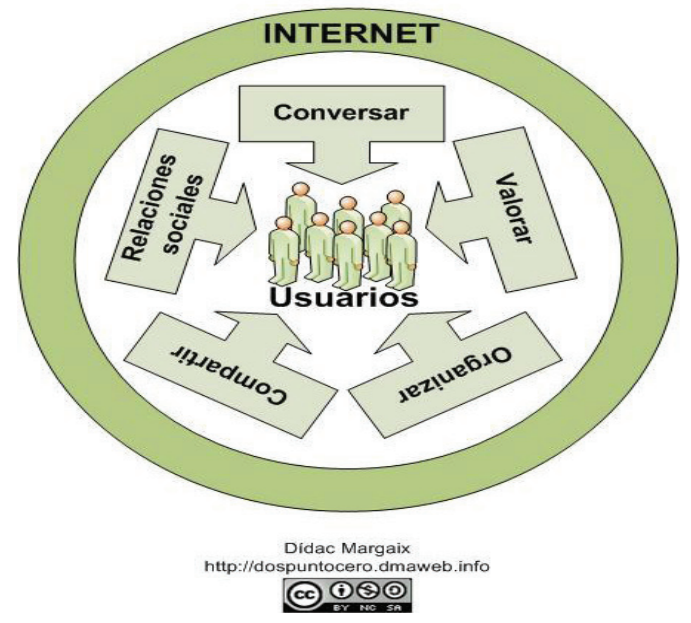

Los principales usos de las redes sociales se exponen gráficamente a continuación.

Figura . Funciones del software social

Nota: Luque, 2008, p. 2.

Como se evidencia, las redes sociales tienen muchos fines positivos o negativos, según las medidas de precaución tomadas al ingresar a ella, por lo tanto, la importancia de fortalecer el pensamiento crítico, especialmente en quienes tienen dificultades para tomar libremente decisiones o se involucran en relaciones riesgosas por el desconocimiento del otro individuo con el cual se interactúa, desarrollando mayor propensión a vivir situaciones que les ponen en peligro, tales como abusos y violaciones sexuales o perdida de sus propias vidas, aspectos los cuales pueden ser abordados dentro de los ejes temáticos vistos en los centros educativos con la finalidad de prevenir y mitigar estas situaciones. 
Ahora bien, el uso de redes sociales muchas veces da paso para que las personas construyan un ideal de sí mismas, dando como resultado la identidad digital, la cual se aborda seguidamente.

\section{Identidad digital}

Es importante recordar que la mayoría del estudiantado en la etapa de la secundaria, a su vez, se encuentran atravesando la adolescencia, entendida por Krauskopf (1997) como:

... un período crucial del ciclo vital en el que los individuos toman una nueva dirección en su desarrollo, alcanzan su madurez sexual, se apoyan en recursos psicológicos y sociales que obtuvieron en su crecimiento previo, asumen para sí las funciones que les permiten elaborar su identidad y plantearse un nuevo proyecto de vida. (p. 23)

Con la construcción de una identidad digital -cuando esta se superpone a la real- se dificulta el desenvolvimiento del estudiante en aspectos claves de su proceso de formación, convirtiéndose en un disociador de su sano aprendizaje y desarrollo, es ahí cuando se debe gestionar desde la educación, estrategias de intervención constructivas del sano desarrollo integral de cada ser humano.

En la etapa de la adolescencia también, se presentan situaciones particulares en los individuos, tales como: su iniciación sexual, cambios en sus relaciones interpersonales y a nivel personal, una búsqueda de independencia. Esto genera que el sujeto se vea expuesto a situaciones de riesgo, en donde se pone a prueba las herramientas adquiridas en etapas previas, las cuales le ayuden a enfrentar problemáticas sociales como la drogadicción, el ingreso a pandillas, el uso inadecuado del tiempo dentro de Internet, en una etapa en la cual, usualmente no se cuenta con la madurez o autonomía necesaria (Papalia, Olds y Feldman, 2005) y en donde es fundamental contar con la guía del profesorado y de personas especialistas en el área de acompañamiento social para lograr superarla exitosamente, mediante la construcción adecuada de la identidad, la cual es necesario que sea congruente con la realidad inmediata de la persona y no con su imaginario.

Ahora bien, el impacto social producido por el uso de las tecnologías de la información, propiamente por las redes sociales, han generado lo que hoy es la llamada identidad digital, definida por Giones y Serrat (2010) como "todo aquello que identifica un individuo en el entorno web personal" (Introducción, párr. 3), se incluye pasatiempos, contactos y los perfiles creados en la red, estos últimos, quizás establecidos de forma falsa, pero siendo convertidos en el ciberespacio como la cédula de identidad, la cual les caracteriza y confiere rasgos único, muchas veces más ficticios que reales, pues suelen ser una proyección de lo que se quiere ser.

También, se habla hoy en día, de una identidad digital personal, definida por Giones y 
Serrat (2010) como:

(...) la habilidad de gestionar con éxito la propia visibilidad, reputación y privacidad en la red como un componente inseparable y fundamental del conjunto de habilidades informacionales y digitales, las cuales se han convertido en fundamentales para vivir en la sociedad informacional. (Introducción, párr. 5)

Dentro de este aspecto se hace importante el trabajo integral y complementario entre todas las disciplinas que componen el eje curricular, buscando desarrollar en el estudiantado, las competencias que le permitan, usar las redes sociales como espacios para satisfacer sus necesidades, desde un ámbito sano y de crecimiento integral para los individuos, mediante la incentivación reflexiva y crítica de las implicaciones tenidas con las conductas y acciones del presente a lo largo de la vida.

Tanto ha sido el crecimiento y aceptación de las redes sociales, dentro de los nuevos estilos de vida, especialmente en aquellos estudiantes, los cuales atraviesan a su vez la etapa de la adolescencia, que la conformación de la identidad digital se superpone muchas veces a la real, como lo expresa García (2010a):

(...) le conceden tanta importancia y le dedican tanto tiempo y atención a lo que se dice de ellos en la Red, o a las fotos o vídeos en los que aparecen. Además, experimentan nuevas formas de relacionarse $y$, muchas veces, expresan emociones y proporcionan información propia o de otras personas, en diversos formatos (texto, audio o vídeo), sin pudor alguno. (p. 20)

Lo mencionado anteriormente, pone muchas veces en peligro al estudiantado, siendo uno de los retos del personal docente conocer cómo están vivenciando sus estudiantes estos espacios, así como el impacto tenido para sus vida y procesos formativos, con el afán de proveerles las herramientas necesarias para su sano desarrollo.

En consecuente, la existencia de la identidad digital, es una realidad en la cual los y las profesionales en educación, deben fortalecer sus mecanismos de intervención en las poblaciones más susceptibles como lo son las personas en la etapa de la adolescencia, quienes suelen ser quienes vivencian más directamente estos cambios tecnológicos, los cuales, han repercutido, tanto positiva como negativamente en la sociedad, generando nuevas necesidades de apoyo en la construcción de la identidad de dicha población, desde todas las disciplinas vinculadas con procesos formativos, enfocando el esfuerzo en el desarrollo de un proyecto de vida, el cual posibilite el camino hacia la autorrealización mediante el desarrollo de la autonomía, conocimientos empíricos y científicos que respalden su actuar.

Por ello, desde la administración de los sistemas educativos, se debe dar "la garantía de 
un aprendizaje vinculado a la formación de la identidad de las personas y de su capacidad para convivir, hacer, emprender y aprender de modo continuo", tal como lo expresa la Comisión Económica para América Latina y el Caribe (CEPAL, s.f., p. 84), siendo ello, parte de los pilares en los cuales se fundamente el actuar de los procesos de formación integral recibidos por el estudiantado, los cuales sirvan como agentes preventivos para el logro adecuado de la conformación de lo que serán el resto de su vida.

Ahora bien, dentro de los aspectos amenazantes para muchos jóvenes, se encuentra el acceso libre a las redes sociales, sin contar con criterios claros para seleccionar sus contactos y establecer posteriormente encuentros con ellos, lo cual genera la necesidad de fortalecer la propia identidad mediante procesos en donde se trabaje la autoestima y sus componentes con el fin de empoderar a esta población.

La creación de una identidad digital, suele responder, en algunos casos, a la renuncia de sí mismo o misma, convergiendo según Giones y Serrat (2010) “... muchos aspectos de carácter sociológico, cultural e incluso psicológico. A veces esta identidad digital no se corresponde con la real, simplemente porque cada cual quiere mostrar lo mejor de sí mismo, con lo que se conoce como self-presentation" (La gestión de la identidad digital, párr. 8). Bajo estas premisas, podría ser que muchos individuos, con perversas intenciones se convierten en un factor amenazante para la persona joven, por lo tanto la necesidad de fortalecer criterios de selección de cuál es el objetivo de participar en las redes sociales y crear un "yo" real, esto mediante el respeto y el cuido de la integralidad individual y colectiva.

Ante ello, desde la educación académica es importante, gestionar políticas de formación integral, en donde se enfaticen los esfuerzos en promocionar estilos de vida saludables, trabajándose de manera conjunta las áreas cognitivas, espirituales, vocacionales, en fin, una visión integral del ser humano. Recodando que la educación incide decisivamente en las oportunidades y la calidad de vida de las personas (CEPAL, s.f.); por ello, la importancia de gestionar procesos de educación holísticos, en donde prive en el estudiantado la autoaceptación y el respeto por sí mismo.

Aunado a lo anterior, es fundamental fomentar esfuerzos, desde las políticas institucionales, por incorporar métodos de enseñanza reflexiva, en donde a partir de los contenidos vistos en clases, se analice la realidad inmediata, sus riesgos, beneficios (García, 2004) y medidas de precaución que se pueden utilizar para frecuentar espacios tan comunes como son las redes sociales y la Internet, y en donde a su vez se conciencie acerca de los cambios propios de la etapa de la adolescencia, así como la búsqueda constante dada en éste período por construir la identidad (Krauskopf, 1997). Producto de las redes sociales, surgen lo que hoy en día se llaman las Ciberrelaciones, las cuales son abordadas seguidamente. 


\section{Ciberrelaciones}

Las formas de iniciar relaciones de pareja o nuevas amistades han cambiado. Hace unos años, lo ideal para iniciar una relación de pareja, era compartir algún tiempo presencial con la otra persona y posteriormente establecer algo más íntimo. Hoy día, la situación ha variado, especialmente en los grupos etarios más jóvenes, pues con la invención de las redes sociales, los procesos de seducción se han modificado, respondiendo en muchos casos, al ingreso de una red social, en la cual se postula un perfil determinado y mediante la utilización de software, se da la conexión con el prototipo de pareja ideal a los gustos y exigencias tenidos, algo que es necesario completar con programas de educación para el amor y la sexualidad, en donde se profundice en la importancia de vivenciar las relaciones humanas y de pareja como una necesidad para alcanzar la satisfacción y plenitud emocional, física y espiritual, aspectos cruciales de una educación integral.

Dentro de las Ciberrelaciones, se pueden distinguir diferentes tipos de relaciones generadas en las redes sociales, están las de tipo amistoso, amoroso y filiales; todas comparten en común un proceso, el cual, consiste usualmente en: contacto inicial, el reconocimiento de un otro como yo y el asentamiento estable de una relación donde se encuentren presentes elementos como la compañía, confidencia y ayuda, situando el inicio de lo que se vendría a configurar como una relación íntima (Roco, 2001).

Dentro la conformación de las ciberrelaciones se suele darla posibilidad de una interacción con la ausencia física del otro individuo, es decir, aquella otra persona que antes había sido excluida de los procesos de interacción y comunicación, donde la presencia de un "otro" no es fundamental, más que en el cibermundo (Roco, 2001), esto muchas veces es un factor peligroso, pues no toda persona que este al otro lado del monitor de una computadora tiene las mejores intenciones para quien comparte aspectos de su vida cotidiana con ella.

Alguna de las relaciones interpersonales que se pueden establecer dentro de las redes sociales, son clasificadas por Levis (2001), como:

- Las relaciones puras de Internet: se desarrollan exclusivamente a través de la computadora, estas se pueden dar, de forma eventual y anónima, mediante canales de chat abiertos, en ellos se puede "charlar" con personas con las que, en general, no se conocen y solo por casualidad, se vuelve a retomar la conversación, se suelen usar disfraces, juegos de personajes y se tiene un margen de conocimiento de que la personas del otro lado puede ser o no quien dice.

Este tipo de relaciones, al permitir adquirir diferentes personalidades, puede favorecer a la comunicación consigo mismo y con sus interlocutores. En este sentido el chat podría verse como un gran espacio de catarsis creativa colectiva. De ellas suelen resultar, amistades y ciberamores. 
- Relaciones "puras" de Internet: se establecen a través de la participación en las innumerables y variadas listas de correo, foros de discusión u otros tipos de comunidades virtuales que existen en la Red. Las características de cada relación cambiarán de acuerdo del contexto en el cual se inició. Muchas veces la relación alcanza una intensidad y nivel de compromiso que, como en el caso de las relaciones iniciadas en un chat, requiere culminar en un encuentro físico.

- Relación en la Red: se mantienen con personas conocidas a través de correo electrónico, programas de mensajería instantánea y canales privados de chat. Cuando estas relaciones son de amor, muchas veces, son acompañadas con la paradójica de ausencia presente del cuerpo de la persona amada y un fantaseado amor perfecto construido desde esa ausencia.

Muchos de las relaciones tenidas mediante el uso de las redes sociales, responden a procesos vinculares sanos, pero otra veces a carencias emocionales y sociales, así como a mandatos patriarcales y culturales, en donde se le da prioridad a la cantidad de relaciones tenidas y no a su calidad, aspecto en lo cual, desde el quehacer de la educación es necesario reflexionar con el fin de construir una sociedad más integral, basada en la búsqueda de satisfacción y de vínculos afectivos menos comercializados e influenciados por el cortoplacismo y la incapacidad de expresar sentimientos y emociones.

La educación tiene como reto, fortalecer en las personas los valores y principios fundamentales que deben regir la conducta humana, mediante acciones de respeto a la gran diversidad de criterios existentes en la conformación de una relación pero siempre buscando el sentido de amor hacia sí mismo y quienes le rodean mediante la conformación de vínculos saludables. También, de acuerdo con la Organización de las Naciones Unidas para la Educación, la Ciencia y la Cultura (UNESCO, s.f.) "tiene la misión de permitir a todos sin excepción hacer fructificar todos sus talentos y todas sus capacidades de creación, lo que implica que cada uno pueda responsabilizarse de sí mismo y realizar su proyecto personal" (p. 12). Todos estos aspectos, remiten una tarea peculiar a la gestión de la educación como agente integral de cambio, el cual es brindar al estudiantado la oportunidad de consolidarse como personas únicas en un espacio y tiempo determinado, para lo cual es fundamental desarrollar la autonomía y la responsabilidad de comprometerse con una toma de decisiones responsable, coherente con el bienestar propio.

\section{Conductas de riesgo en la adolescencia}

La adolescencia, como se vio anteriormente, es una etapa muy particular, en donde se caracteriza por la heterogeneidad de los individuos, además de la constante construcción de su identidad, situación que se debe tomar en cuenta dentro de los procesos de educación en la conformación de estrategias adecuadas para esta población, concordando. Al respecto, Gil y Romo (2008) concuerdan en que: 
(...) si se quiere comprender por qué una persona asume ciertos riesgos, habrá que tener en cuenta, que ello es más producto de la interacción social que de una decisión individual. De ahí la necesidad de plantear un cambio en la unidad de análisis, del individuo a las relaciones e interacciones sociales y la comprensión de las distintas conductas de riesgo de manera conjunta. (p. 494)

Lo anterior, son aspectos fundamentales de ser trabajados en los centros educativos y la familia, como instituciones complementarias estrechamente vinculadas con la educación de las personas, siendo necesario el surgimiento de una comunión armoniosa entre ambas, enfocada explícitamente en nutrir las bases educativas de sus integrantes en todos los aspectos de la vida cotidiana.

Cuando desde las instituciones educativas se realizan esfuerzos por concienciar a las personas jóvenes de las conductas de riesgo que se pueden asumir dentro de las redes sociales, es probable que desarrollen más herramientas para navegar dentro de ellas, pues se tiene conocimiento de las implicaciones tenidas para su vida. Se recalca como esta población, al estar en una fase importante de la construcción de su identidad, muchas veces utilizan casi de manera única y exclusiva las redes sociales para fomentar sus relaciones interpersonales, por dificultades para hacerlo de manera inmediata en su contexto, teniendo mayor propensión a experimentar conductas, las cuales afectan su desarrollo e integralidad, aspectos que no pueden ser erradicados totalmente desde las aulas educativas, pero si amortiguar su impacto, si desde aspectos como la lectura de grupo se trabaja para complementar lo individual con lo colectivo, en una diada de la cual se puede sacar gran provecho para disminuir éste tipo de conductas.

Las conductas de riesgo, según Berra y Dueñas (2008):

(...) pueden ser diferentes entre sí, pero su función psicológica en los adolescentes suele ser similar. Con ella los adolescentes pretenden expresar el rechazo a las normas convencionales, su independencia ante el control paterno, alcanzar un rol prestigioso con el grupo de amigos, que en algunas ocasiones expresa una adaptación pasiva a una sociedad de consumo, que define a las personas por el "tener" (cosas, poder, relaciones) más que en el "ser" una mejor persona humana, moldeando a través de los medios masivos de información (TV, Internet...) estilos de vida no saludables. (p. 120)

Es importante evidenciar, la probabilidad de que muchos de los adolescentes que se encuentran en los salones de clases, pueden estar vivenciando conductas de riesgo dentro de sus acciones cotidianas; por ello, la educación no se puede aislar de esta realidad, por lo cual, la importancia de su abordaje preventivo y constante dentro de los ejes transversales incorporados a los contenidos académicos vistos en clases, como parte del análisis de la realidad.

Cabe destacar que la Internet, y más recientemente, las redes sociales, se permutan -casi 
de forma incontrolable- con los procesos de enseñanza y aprendizaje desarrollados en las aulas, potenciando factores de riesgo y beneficio según el abordaje dado por los profesionales en educación, quienes enfrentan el reto de mantenerse dentro de las nuevas corrientes tecnológicas, siendo un soporte para formar personas sapientes en todo lo amplio del término, en aspectos de pensamiento crítico para reconocer y apropiarse de las herramientas necesarias para enfrentar los retos de esta nueva era digital.

A continuación, se presentan las principales conductas de riesgo, mencionadas por la Fundación Paniamor (2009), vivenciadas por las personas tras el uso inadecuado de las redes sociales, las cuales, es fundamental que sean conocidas por profesionales en educación con la finalidad de detectarlas en la población estudiantil y de esta manera, prevenir o amortiguar sus repercusiones. Estas son:

- Pornografía con personas menores de edad.

- Robo y fraude virtual.

- Morphing: uso y alteración de imágenes con fines de burla o acoso. Es un efecto especial utilizado para modificar el rostro de las personas hasta transformarlas en el de otras.

- Exposición a contenido no deseado.

- Ciberbullying: intimidación o matonismo virtual. Corresponde a la utilización de las "posibilidades que ofrece la tecnología digital para que un individuo o grupo las use deliberadamente y de manera repetida para el acoso o amenaza hacia otro individuo o grupo mediante el envío o publicación de contenidos en cualquier formato..." (García, 2010b, p. 58).

- Grooming: preparación, manipulación y chantaje en línea con fines sexuales. Corresponde al "conjunto de estrategias que una persona adulta desarrolló para ganarse la confianza a través de Internet con el fin último de obtener concesiones de índole sexual" (Álvarez, 2009, p. 7).

- Flaming: uso de lenguaje vulgar y agresivo. Es la interacción hostil e insultante en Internet.

- Spamming: correos basura con información no solicitada.

- Solicitación sexual: solicitud explícita en línea para cometer actos sexuales.

- Sexting: intercambio de imágenes personales con contenido sexual.

Como se evidencia, son muchos los riesgos a los cuales se es susceptible dentro de las redes sociales, por ello, se hace importante que los profesionales y las profesionales en educación, los conozcan para de esta manera, desarrollar estrategias de información y se concientice al estudiantado en la necesidad del desarrollo del pensamiento crítico-analítico, en aras de posibilitar procesos adecuados de toma de decisiones en los cuales, el individuo logre alcanzar su bienestar integral. 
Por los cambios que acontecen con el uso de las redes sociales, en la forma en cómo interactúan las nuevas generaciones, es necesario que desde el sistema educativo costarricense se gestionen esfuerzos para prevenir conductas de riesgo vinculadas a las acciones cometidas en estos espacios con la finalidad de propiciar en los y las adolescentes las herramientas necesarias para que se fortalezcan integralmente, logrando de esta manera mayor calidad de vida.

\section{Conclusiones}

Es evidente el aumento en la cantidad de personas que ingresan a las redes sociales y se ven afectadas tanto positiva como negativamente por ellas, en esto se fundamenta la importancia de trabajar la temática en los salones educativos para fortalecer el pensamiento crítico-analítico en la población estudiantil adolescente, con el fin de ayudar en la construcción de su identidad y de un adecuado proyecto de vida.

Existen múltiples y variados factores de riesgo a los cuales se debe enfrentar la población estudiantil adolescente tras el uso de la Internet y las redes sociales, por lo cual, desde todas las disciplinas que componen el currículo académico, se hace fundamental el trabajo conjunto, con el fin de fortalecer el conocimiento y desarrollo de estrategias, las cuales, nutran al estudiantado de las herramientas básicas para enfrentar los retos y desafíos presentados en estos sitios con la finalidad de que puedan salir airosos a las situaciones presentadas en ellos.

Es fundamental desarrollar competencias y habilidades sociales en las personas adolescentes, mediante procesos de empoderamiento y de desarrollo adecuado de la autoestima, con la finalidad de fortalecer sus capacidad para enfrentar los cambios sociales vividos con el uso de la Internet y las redes sociales, por lo cual, la educación debe ser integral y principalmente, acorde con los cambios vividos con la nueva era tecnológica de la cual se es partícipe.

En el proceso de construcción de la identidad vivido en la adolescencia, se hace necesario potenciar mediante la discusión crítica de la realidad social, en aspectos tales como el uso adecuado de la Internet y las redes sociales, con el fin de lograr la congruencia entre el querer y el deber, mediante el amor y el respeto propio, con el fin de favorecer el desarrollo integral.

El y la profesional en gestión de la educación debe asumir un liderazgo especial en el desarrollo de procesos formativos integrales, congruentes con la realidad social y tecnológica vivida, en donde se busque desarrollar en el estudiantado los criterios suficientes para enfrentar los retos y desafíos propiciados con el acceso a Internet, así como a las diferentes redes sociales para lograr su sano desarrollo cognitivo, emocional, espiritual con el fin de que se trascienda en su formación, educando para la vida y no meramente para una parte de ella. 


\section{Referencias}

Álvarez, G. (2009). Cómo protegernos de los peligros de Internet. Madrid, España: CATARATA.

Bernárdez, A. (2006). A la búsqueda de una "habitación propia": comportamiento de género en el uso de Internet y los chats en la adolescencia. Revista de Estudios de la Juventud, (73), pp. 69-82. Recuperado en: http://eprints.ucm.es/10410/

Berra, M. y Dueñas, R. (2008). Educación para la salud: conductas de riesgo en los adolescentes y jóvenes. Revista Científica Electrónica de Psicología, (5). Recuperado en: http://dgsa.uaeh. edu.mx/revista/psicologia/IMG/pdf/7_-_No._5.pdf

Comisión Económica para América Latina y el Caribe (CEPAL). (s.f.). La educación como eje promotor de desarrollo humano. Recuperado en: http://www.cepal.org/publicaciones/ xml/1/21541/capitulo3.pdf

Fernández, B. (2010). Las redes sociales. Lo que hacen sus hijos en Internet. España: Editorial Club Universitario.

Fundación Paniamor. (2009). Nuevas expresiones de violencia interpersonal y social en el ciberespacio desde la vivencia adolescente. Recuperado en: http://www.dgmme.sep.gob.mx/archivos/ Informe_Costa_Rica.pdf

García, F. (2010a). Interneten la vida de nuestros hijos ¿Cómo transformar los riesgos en oportunidades? Foro Generaciones Interactivas. Recuperado en: http://www.generacionesinteractivas.org/ upload/libros/Internet-en-la-vida-de-nuestros-hijos.pdf

García, F. (2010b).Las redes sociales en la vida de tus hij@s.Foro Generaciones Interactivas. Recuperado en:http://www.generacionesinteractivas.org/upload/libros/Las-Redes-Sociales-en-la-vidade-tus-hij@s.pdf

García, J. (2004). La administración y gestión educativa: algunas lecciones que nos deja su evolución en los Estados Unidos y México. Recuperado en: http://tumbi.crefal.edu.mx/rieda/images/ rieda-2004-1/articulo_ensayo1.pdf 
Gil, E. y Romo, N. (2008). Conductas de riesgo en adolescentes urbanos Andaluces. Revista Miscelánea Comillas, 66 (129), pp. 493-509. Recuperado en: http://www.ugr.es/ esmujer/ Paginas\%20Personales/EugeniaGil/pdf/ConductasRiesgoadolescentesandaluces.pdf

Giones, A. y Serrat, M. (2010). La gestión de la identidad digital: una nueva habilidad informacional y digital. Revista TéxtosUniversitaris de Biblioteconomía i documentació. Recuperado en: http://www.ub.edu/bid/24/pdf/giones2.pdf

Instituto Nacional de Estadística y Censo (INEC). (2010). Uno de cada cinco ticos usa las redes sociales. Recuperado en:http://www.unimercentroamerica.com/biblioteca_virtual/Redes_ Sociales.pdf

Juncar, J. (2001). Internet. Barcelona, España: MARCOMBO S.A.

Krauskopf, D. (1997). Adolescencia y educación. San José, Costa Rica: EUNED.

Levis, D. (2001). Relaciones afectivas en Internet: Buscando salir del círculo de la soledad. Revista de la Facultad de Ciencias Sociales, Universidad Nacional de Costa Rica. ABRA. Recuperado en: http://www.diegolevis.com.ar/seccionebs/Articulos/amorenred.pdf

Luque, F. (2008). ¿Qué podemos hacer en una red social? Recuperado en: http://ciberespiral.net/ webje08/que_podemos_hacer.pdf

Marambio, C. (s.f.). Gestión para una educación de calidad en un mundo global. EDUMAR. Recuperado en: http://www.edumar.cl/documentos/gestion.pdf

Moral, F. (2001). Aspectos psicosociales de la comunicación y de las relaciones personales en Internet. Anuario de Psicología, 32 (2), pp. 13-30. Recuperado en: http://www.raco.cat/index. php/anuariopsicologia/article/viewFile/61665/96249

Organización de las Naciones Unidas para la Educación, la Ciencia y la Cultura (UNESCO). (s.f.). La educación encierra un tesoro. Recuperado en: http://www.unesco.org/education/pdf/ DELORS_S.PDF

Papalia, D.; Olds, S. y Feldman, R. (2005). Desarrollo Humano. México, DF: McGraw-Hill/ Interamericana Editores, S.A. 
Roco, G. (2001). Un estudio del establecimiento de relaciones íntimas mediadas por computador. El caso de IRC en Internet. Revista TEXTOS de la CiberSociedad, (1). Recuperado en: http:// www.cibersociedad.net

Sander, B. (s.f.). Nuevas tendencias de la gestión educativa: democracia y calidad. Recuperado en: http://www.educando.edu.do/files/4313/4643/1519/NUEVAS_TENDENCIAS_EN_LA_ GESTIN_EDUCATIVA.pdf 